\title{
A COUNTEREXAMPLE TO THE UNIMODULAR CONJECTURE ON FINITELY GENERATED DIMENSION GROUPS
}

\author{
NORBERT RIEDEL
}

\begin{abstract}
We give a series of examples of simple finitely generated dimension
\end{abstract} groups which cannot be obtained as the inductive limit of a system

$$
\mathbf{z}^{r} \stackrel{A_{1}}{\rightarrow} \mathbf{z}^{A_{2}} \rightarrow \cdots \mathbf{z}^{\boldsymbol{A}_{n}} \rightarrow \ldots,
$$

where each $A_{n}$ is a unimodular matrix whose entries are nonnegative integers.

1. In this note we are concerned with ordered groups $G$ of the following form. $G$ is equal to $\mathbf{Z}^{r}$ as an abelian group only, for some $r \in \mathbf{N}$, and there exists a set $\left\{a^{(1)}, \ldots, a^{(p)}\right\}$ of linearly independent vectors in $\left(\mathbf{R}^{r}\right)^{+}$such that the positive cone $G^{+}$of $G$ is given by

$$
G^{+}=\left\{z \in \mathbf{Z}^{r} /\left\langle a^{(i)}, z\right\rangle>0 ; i=1, \ldots, p\right\} \cup\{0\} .
$$

$G$ is a dimension group if it satisfies the Riesz interpolation property (for the definitions concerning dimension groups we refer to [1], [2]). Effros and Shen conjectured in [3] (see also [1]) that if $G$ is a dimension group, then there exists a sequence $A_{1}, A_{2}, \ldots$ in the set $\mathrm{GL}(r, \mathrm{Z})^{+}$of all unimodular matrices whose entries are nonnegative integers, such that

$$
G^{+}=\bigcup_{n=1}^{\infty}\left(A_{n} \ldots A_{1}\right)^{-1}\left(\mathbf{Z}^{r}\right)^{+} .
$$

In [4] we have shown that the conjecture is true if $G$ is simple (i.e. $G^{+}$has no nontrivial faces) and $p=1$ holds. Using the theory of diophantine approximation we will show in the sequel that for $p=r-1(r>3)$ there exist simple dimension groups for which the conjecture of Effros and Shen is false. If $p=r-1$ holds then we can use the following criterion in order to decide whether $G$ is a simple dimension group or not. Let $b$ be a nonzero vector which is orthogonal to the hyperplane in $\mathbf{R}^{r}$ which is generated by the vectors $a^{(1)}, \ldots, a^{(r-1)}$.

1.1. Proposition [2, Corollary 4.2]. The following statements are equivalent.

(1.1.1) $G$ is a simple dimension group.

(1.1.2) $\operatorname{det}\left(a^{(1)}, \ldots, a^{(r-1)}, z\right) \neq 0$ holds for any nonzero vector $z$ in $\mathbf{Z}^{r}$.

(1.1.3) The components of the vector $b$ are linearly independent over the rational field Q.

Received by the editors October 23, 1980 and, in revised form, January 5, 1981.

1980 Mathematics Subject Classification. Primary O6F20, 10F10, 46L99.

(C) 1981 American Mathematical Society 0002-9939/81/0000-0402/\$02.25 
2. Henceforth we fix $r \geqslant 3$. Let $a^{(1)}, \ldots, a^{(r-1)}$ be fixed linearly independent vectors in $\left(\mathbf{R}^{r}\right)^{+}$such that the ordered group $G$ defined above is a simple dimension group. Denote by $b$ the unique vector in $\mathbf{R}^{r}$ such that

$$
\langle b, x\rangle=\operatorname{det}\left(a^{(1)}, \ldots, a^{(r-1)}, x\right) .
$$

By multiplying one of the vectors $a^{(1)}, \ldots, a^{(r-1)}$ with a suitable constant we may assume that the last component $b_{r}$ of $b$ is equal to 1 . Of course, this does not impair the definition of $G$.

We need some other notations. For any subset $M \subseteq \mathbf{R}^{r}$ we denote by $\operatorname{conv}(M)$ the convex hull of $M$. For any vector $x=\left(x_{1}, \ldots, x_{r}\right)^{t} \in \mathbf{R}^{r} \backslash\{0\}$ we set $\bar{x}=$ $\|x\|^{-1} x$, where $\|x\|$ is the $l^{1}$-norm of $x$, and we set $\check{x}=\left(x_{1}, \ldots, x_{r-1}\right)^{t}$.

Now we assume that $A_{1}, A_{2}, \ldots$ is a sequence in $\mathrm{GL}(r, \mathrm{Z})^{+}$such that

$$
G^{+}=\bigcup_{n=1}^{\infty}\left(A_{n} \ldots A_{1}\right)^{-1}\left(\mathbf{Z}^{r}\right)^{+} \text {, }
$$

or equivalently,

$$
\operatorname{conv}\left(\left\{\bar{a}^{(1)}, \ldots, \bar{a}^{(r-1)}\right\}\right)=\bigcap_{n=1}^{\infty}\left\{\bar{x} / x \in \operatorname{conv}\left(A_{1}^{t} \ldots A_{n}^{t}\left(\mathbf{Z}^{r}\right)^{+}\right) \backslash\{0\}\right\} .
$$

2.1. Definition [5, II, \$4]. To each vector $x \in \mathbf{R}^{r-1}$ we associate a linear form $L_{x}$ on $\mathbf{Z}^{r-1}$ by $L_{x}(z)=\langle x, z\rangle, z \in \mathbf{Z}^{r-1} . L_{x}$ is called badly approximable if there exists a positive constant $\alpha$ such that $\left|L_{x}(z)-q\right| \geqslant \alpha\|z\|_{\infty}^{-r+1}$ holds for each $z \in \mathbf{Z}^{r-1} \backslash\{0\}, q \in \mathbf{Z}$, where $\|z\|_{\infty}$ denotes the maximum norm of $z$.

Our main purpose in this section is to show that in our present situation the linear form $L_{\breve{b}}$, as defined above, can not be badly approximable.

We need the following lemma whose easy proof is left to the reader.

2.2. LEMMA. Let $\Delta_{1}, \Delta_{2}, \ldots$ be a monotonely decreasing sequence of $r-1$ dimensional simplices in $\mathbf{R}^{r}$ and let $\Delta=\bigcap_{n=1}^{\infty} \Delta_{n}$. Moreover let $v^{(1, n)}, \ldots, v^{(r, n)}$ be the extreme points of $\Delta_{n}$. Then there exists a monotonely increasing sequence $n_{1}, n_{2}, \ldots$ of positive integers such that $\left\{v^{\left(j, n_{i}\right)}\right\}_{i \in \mathbf{N}}$ converges to a point in $\Delta$ for each $j$, $1 \leqslant j \leqslant r$, and for any extreme point $w$ in $\Delta$ there exists $a j, 1<j<r$, such that $w=\lim _{i \rightarrow \infty} v^{\left(j, n_{i}\right)}$.

It follows from (2.1) together with 2.2 that there exists a monotonely increasing sequence $n_{1}, n_{2}, \ldots$ of positive integers, a permutation 6 of the set of integers $\{1, \ldots, r\}$, and a point $w$ in $\operatorname{conv}\left(\left\{\bar{a}^{(1)}, \ldots, \bar{a}^{(r-1)}\right\}\right)$ such that, if $v^{(6(1), i)}, \ldots, v^{(6(r), i)}$ are the column vectors of the matrix $B^{(i)}=A_{1}^{t} \ldots A_{n_{i}}^{t}$, then we have

$$
\lim _{i \rightarrow \infty} \bar{v}^{(j, i)}=\bar{a}^{(i)} \quad \text { for } 1 \leqslant j \leqslant r-1 ; \quad \lim _{i \rightarrow \infty} \bar{v}^{(r, i)}=w .
$$

Now we can prove the following proposition which is crucial for the proof of Theorem 2.4.

2.3. Proposition. There exists $a k \in\{1, \ldots, r-1\}$ such that

$$
\lim _{n \rightarrow \infty} \operatorname{det}\left(\bar{v}^{(1, n)}, \ldots, \bar{v}^{(r, n)}\right)^{-1} \operatorname{det}\left(\bar{a}^{(1)}, \ldots, \bar{a}^{(r-1)}, \bar{v}^{(k, n)}\right)=0 .
$$


Proof. There exists a $k \in\{1, \ldots, r-1\}$ such that $\vec{a}^{(k)} \neq w$. Since $\operatorname{conv}\left(\left\{\bar{a}^{(1)}, \ldots, \bar{a}^{(r-1)}\right\}\right)$ is a simplex containing $w$, we have

$$
\bar{a}^{(k)} \notin M=\operatorname{conv}\left(\left\{\bar{a}^{(j)} / j \neq k\right\} \cup\{w\}\right) \text {. }
$$

It follows that

$$
\delta=\inf \left\{\left\|\bar{a}^{(k)}-x\right\| / x \in M\right\}>0 .
$$

Since $\bar{a}^{(k)}$ is contained in $\operatorname{conv}\left(\left\{\vec{v}^{(1, n)}, \ldots, \vec{v}^{(r, n)}\right\}\right)$ for each $n \in \mathbf{N}$, we can write

$$
\bar{a}^{(k)}=\sum_{j=1}^{r} t_{j}^{(n)} \bar{v}^{(j, n)},
$$

with $t_{j}^{(n)} \in[0,1]$ and $\Sigma_{j=1}^{r} t_{j}^{(n)}=1$. By our assumption $G$ is a simple dimension group. Therefore we obtain from (1.1.2) that $\operatorname{det}\left(\bar{a}^{(1)}, \ldots, \bar{a}^{(r-1)}, \bar{v}^{(k, n)}\right) \neq 0$. In particular $\bar{v}^{(k, n)} \neq \bar{a}^{(k)}$ and $t_{k}^{(n)} \neq 1$. Thus we can define

$$
\lambda_{j}^{(n)}=t_{j}^{(n)}\left(1-t_{k}^{(n)}\right)^{-1}, \quad u^{(n)}=\sum_{j \neq k} \lambda_{j}^{(n)} \bar{v}^{(j, n)} .
$$

Since $\left\{\bar{v}^{(j, n)}\right\}_{n \in N}$ converges to $\bar{a}^{(j)}$ for $1<j<r-1$, and to $w$ for $j=r$, there exists a $n_{0} \in \mathbf{N}$ such that for any $n \geqslant n_{0}$

$$
\left\|\bar{v}^{(j, n)}-\bar{a}^{(j)}\right\|<\delta / 2 \text { for } j \neq r ; \quad\left\|\bar{v}^{(r, n)}-w\right\|<\delta / 2 .
$$

If we set

$$
\tilde{u}^{(n)}=\sum_{j \neq k, r} \lambda_{j}^{(n)} \bar{a}^{(j)}+\lambda_{r}^{(n)} w
$$

then we obtain for each $n>n_{0}$

$$
\left\|u^{(n)}-\tilde{u}^{(n)}\right\|<\delta / 2 .
$$

Since $\tilde{u}^{(n)}$ is contained in $M$ we have

$$
\left\|\tilde{u}^{(n)}-\bar{a}^{(k)}\right\|>\delta .
$$

By combining the last two inequalities we obtain an estimation for the distance of $u^{(n)}$ and $\bar{a}^{(k)}$ :

$$
\left\|u^{(n)}-\bar{a}^{(k)}\right\|>\delta / 2 \text { for } n \geqslant n_{0} .
$$

Since $\bar{a}^{(1)}, \ldots, \bar{a}^{(r-1)}, u^{(n)}$ are contained in $\operatorname{conv}\left(\left\{\bar{v}^{(1, n)}, \ldots, \bar{v}^{(1, r)}\right\}\right)$ we have

$$
\left|\operatorname{det}\left(\bar{a}^{(1)}, \ldots, \bar{a}^{(r-1)}, u^{(n)}\right)\right|<\left|\operatorname{det}\left(\bar{v}^{(1, n)}, \ldots, \bar{v}^{(r, n)}\right)\right| \text {. }
$$

Therefore we obtain for each $n \geqslant n_{0}$

$$
\begin{aligned}
& \frac{\left|\operatorname{det}\left(\bar{a}^{(1)}, \ldots, \bar{a}^{(r-1)}, \bar{v}^{(k, n)}\right)\right|}{\left|\operatorname{det}\left(\bar{v}^{(1, n)}, \ldots, \bar{v}^{(r, n)}\right)\right|} \\
& \quad<\frac{\left|\operatorname{det}\left(\bar{a}^{(1)}, \ldots, \bar{a}^{(r-1)}, t_{k}^{(n)^{-1}}\left(\bar{a}^{(k)}-u^{(n)}\right)+u^{(n)}\right)\right|}{\left|\operatorname{det}\left(\bar{a}^{(1)}, \ldots, \bar{a}^{(r-1)}, u^{(n)}\right)\right|}=\left|1-t_{k}^{(n)}{ }^{-1}\right| .
\end{aligned}
$$

Since $\left\|u^{(n)}-\bar{a}^{(k)}\right\|>\delta / 2$ holds for each $n \geqslant n_{0}$ and since

$$
\left\|\bar{a}^{(k)}-\bar{v}^{(k, n)}\right\|=\left|1-t_{k}^{(n)^{-1}}\right|\left\|u^{(n)}-\bar{a}^{(k)}\right\|
$$


converges to zero for $n \rightarrow \infty$ it follows that $\left|1-t_{k}^{(n)^{-1}}\right|$ converges to zero. Hence

$$
\lim _{n \rightarrow \infty} \operatorname{det}\left(\bar{v}^{(1, n)}, \ldots, \bar{v}^{(r, n)}\right)^{-1} \operatorname{det}\left(\bar{a}^{(1)}, \ldots, \bar{a}^{(r-1)}, \bar{v}^{(k, n)}\right)=0 .
$$

2.4. THEOREM. In the situation considered above the linear form $L_{b}$ is not badly approximable.

Proof. Suppose that our assertion is not true. Then there exists a positive constant $\alpha$ such that

$$
|\langle b, z\rangle| \geqslant \alpha\|\check{z}\|_{\infty}^{-r+1} \text { for each } z \in \mathbf{Z}^{r} \backslash\{0\} .
$$

First we show the following.

(2.4.2) There exists a positive constant $\beta$ such that

$$
\left\|v^{(i, n)}\right\|\left\|v^{(j, n)}\right\|^{-1}<\beta \quad \text { for each } n \in \mathrm{N} ; \quad i, j \in\{1, \ldots, r\} .
$$

Suppose that this is not true. Let $k_{n}=\min \left\{\left\|v^{(j, n)}\right\| / 1<j<r\right\}$ and $l_{n}=$ $\left\|v^{(1, n)}\right\|\left\|v^{(2, n)}\right\| \cdots\left\|v^{(r, n)}\right\|$. Then there exists a monotonely increasing sequence $n_{1}, n_{2}, \ldots$ of positive integers such that $\lim _{i \rightarrow \infty} k_{n_{1}}^{r} l_{n_{i}}^{-1}=0$. We can find an $m \in\{1, \ldots, r\}$ such that $\left\|v^{\left(m, m_{i}\right)}\right\|=k_{m_{i}}$ for infinitely many $i \in \mathbf{N}$. Thus we may choose the sequence $n_{1}, n_{2}, \ldots$ in such a manner that in addition $\left\|v^{\left(m, n_{1}\right)}\right\|=k_{n_{1}}$ holds for each $i \in \mathbf{N}$. We set $d=\left\|a^{(1)}\right\| \cdots\left\|a^{(r-1)}\right\|$. Now we obtain from the inequality

$$
\left|\operatorname{det}\left(\bar{a}^{(1)}, \ldots, \bar{a}^{(r-1)}, \bar{v}^{(m, n)}\right)\right|<\left|\operatorname{det}\left(\bar{v}^{(1, n)}, \ldots, \bar{v}^{(r, n)}\right)\right|,
$$

which is true for any $n \in \mathbf{N}$,

$$
\begin{aligned}
k_{n_{i}}^{r-1}\left|\operatorname{det}\left(a^{(1)}, \ldots, a^{(r-1)}, v^{\left(m, n_{i}\right)}\right)\right| & =k_{n_{i}}^{r} d^{-1}\left|\operatorname{det}\left(\bar{a}^{(1)}, \ldots, \bar{a}^{(r-1)}, \bar{v}^{\left(m, n_{i}\right)}\right)\right| \\
& <k_{n_{i}}^{r} d^{-1}\left|\operatorname{det}\left(\bar{v}^{\left(1, n_{i}\right)}, \ldots, \bar{v}^{\left(r, n_{i}\right)}\right)\right| \\
& =k_{n_{i}}^{r} l_{n_{i}}^{-1} d^{-1}\left|\operatorname{det}\left(v^{\left(1, n_{i}\right)}, \ldots, v^{\left(r, n_{i}\right)}\right)\right|=k_{n_{i}}^{r} l_{n_{i}}^{-1} d^{-1} .
\end{aligned}
$$

From this we infer that

$$
\lim _{i \rightarrow \infty}\left\|v^{\left(m, n_{i}\right)}\right\|^{r-1}\left|\operatorname{det}\left(a^{(1)}, \ldots, a^{(r-1)}, v^{\left(m, n_{i}\right)}\right)\right|=0 .
$$

Since $\left\|\check{v}^{\left(m, n_{i}\right)}\right\|_{\infty}<\left\|v^{\left(m, n_{i}\right)}\right\|$ holds for each $i \in \mathbf{N}$, this implies

$$
\lim _{i \rightarrow \infty}\left\|\check{v}^{\left(m, n_{i}\right)}\right\|_{\infty}^{r-1}\left|\left\langle b, v^{\left(m, n_{i}\right)}\right\rangle\right|=0 .
$$

However, this contradicts (2.4.1).

Using 2.3 as well as (2.4.2) we can now easily complete the proof of our theorem. Let $k \in\{1, \ldots, r-1\}$ be chosen as in 2.3. By (2.4.2) there exists a positive constant $\beta$ such that

$$
\left\|v^{(k, n)}\right\|^{r}<\beta\left\|v^{(1, n)}\right\| \cdots\left\|v^{(r, n)}\right\| \quad \text { for any } n \in \mathrm{N} \text {. }
$$

Since $\left|\operatorname{det}\left(B_{n}\right)\right|=1$ holds for each $n \in \mathbf{N}$, we obtain now

$$
\begin{aligned}
& \beta\left|\operatorname{det}\left(\bar{a}^{(1)}, \ldots, \bar{a}^{(r-1)}, \bar{v}^{(k, n)}\right)\right|\left|\operatorname{det}\left(\bar{v}^{(1, n)}, \ldots, \bar{v}^{(r, n)}\right)\right|^{-1} \\
&>\left\|v^{(k, n)}\right\|^{r-1}\left|\operatorname{det}\left(\bar{a}^{(1)}, \ldots, \bar{a}^{(r-1)}, v^{(k, n)}\right)\right| \\
&=\left\|v^{(k, n)}\right\|^{r-1} d^{-1}\left|\operatorname{det}\left(a^{(1)}, \ldots, a^{(r-1)}, v^{(k, n)}\right)\right| .
\end{aligned}
$$


Therefore, by 2.3 ,

$$
\lim _{n \rightarrow \infty}\left\|\check{v}^{(k, n)}\right\|_{\infty}^{r-1}\left|\left\langle b, v^{(k, n)}\right\rangle\right|=0
$$

and this contradicts (2.4.1).

3. By using the results of $\$ 2$ we are now able to construct a lot of counterexamples to the unimodular conjecture. Let $x$ be a vector in $\mathbf{R}^{r-1}$ such that $L_{x}$ is badly approximable. By [5, II, §4, Theorem 4A] we may choose $x$ in such a manner that the components $x_{1}, \ldots, x_{r-1}$ of $x$ lie in an algebraic number field $K$ of degree $r$ and $\left\{1, x_{1}, \ldots, x_{r-1}\right\}$ is a basis of $K$. We also demand that the components of $x$ do not all have a positive sign. (If necessary, change the basis in $\mathbf{Z}^{r-1}$.) We set $b=\left(x_{1}, \ldots, x_{r-1}\right)^{t}$, and we choose $r-1$ linearly independent positive vectors in the hyperplane which is orthogonal to $b$. (Since the components of $x$ do not all have a positive sign this can always be done.) Now we define a simple dimension group $G$ as in $\S 1$. In order to show that $G$ cannot be obtained as the inductive limit of a unimodular system of groups it is necessary to state that the property that $b$ gives rise to a badly approximable linear form $L_{b}$ is left invariant under isomorphisms of the group $G$. To speak more precisely, if $G^{\prime}$ is an isomorphic copy of $G$ and we associate a vector $b^{\prime}$ with $G^{\prime}$ in the same manner as the vector $b$ with $G$, then we have $\gamma b^{\prime}=A b$ for some $A \in \mathrm{GL}(r, \mathrm{Z})$ and a nonzero constant $\gamma$. Now some simple calculations show that $L_{b^{\prime}}$ is also badly approximable. Thus, by an application of 2.4 , we can conclude that $G$ is not isomorphic to the inductive limit of a system $\mathbf{Z}^{r} \stackrel{A_{1}}{\rightarrow} \mathbf{Z}^{r} \stackrel{A_{2}}{\rightarrow} \cdots \mathbf{Z}^{r} \stackrel{A_{n}}{\rightarrow} \ldots$ with $A_{n} \in \mathrm{GL}(r, \mathbf{Z})^{+}$for each $n \in \mathbf{N}$.

It follows from a theorem of Khintchine (see [5, III, §3, Theorem 3A]) together with a transference principle for badly approximable linear forms (see [5, IV, §5, Theorem 5B]) that the set of all vectors $x \in \mathbf{R}^{r-1}$ such that $L_{x}$ is badly approximable has Lebesgue measure zero. Thus there is still some hope that the conjecture of Effros and Shen is valid for a rather big class of finitely generated dimension groups.

\section{REFERENCES}

1. E. G. Effros, Dimensions and $C^{*}$-algebras, Lecture Notes, UCLA, August, 1980.

2. E. G. Effros, D. E. Handelman and C. L. Shen, Dimension groups and their affine representations, Amer. J. Math. 102 (1980), 385-407.

3. E. G. Effros and C. L. Shen, Dimension groups and finite difference equations, J. Operator Theory 2 (1979), 215-231.

4. N. Riedel, Classification of dimension groups and iterating systems, Math. Scand. 48 (1981).

5. W. M. Schmidt, Diophantine approximation, Lecture Notes in Math., vol. 785, Springer-Verlag, Berlin and New York, 1980.

Institut für Mathematik, Technische Universität, D-8000 München, Federal Republc of GRRMANY 